\title{
ABSTRACTS
}

\section{Canadian Consortium on Neurodegeneration in Aging (CCNA) Partners Forum and Science Days 2021: Abstracts from the trainee poster competition

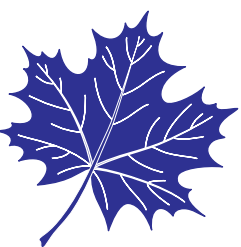

COLLABORATION FOR CONNECTIVITY ; OCTOBER 12-15, 2021

https://doi.org/10.5770/cgj.25.596

\section{CATEGORY: INDIGENOUS \\ FOCUSED, COMMUNITY-ENGAGED RESEARCH PROJECT}

\section{Consensus Building approach in Indigenous Context}

Nabina Sharma ${ }^{1}$, Jennifer Walker ${ }^{2} .{ }^{1}$ Laurentian University; ${ }^{2}$ McMaster University.

Abstract: Consensus methods have been used in health care for a long time to reach agreement among experts when there is a lack of information or conflicting information on a health topic. The Delphi and nominal group techniques are extensively used in health research. Although both consensus methods are transparent in developing health research agendas, their emphasis on clinical and academic experts is problematic in Indigenous research. Another consensus approach named Glaser's state-of-the-art is being used in Indigenous research. In this approach, a panel of experts identifies additional experts who collectively engage in iterative rounds to develop a consensus statement based on current research. We will be using a modified Glaser's state-of-the-art approach to develop an informant-based functional assessment tool to assess the instrumental activities of daily living in people living with dementia. In the first phase, we will form a core research team, set up an Indigenous community advisory group (CAG), and conduct a focus group with health professionals and in-depth interviews with caregivers to develop a draft functional assessment tool. In the second phase, we will refine the tool using a consensus-building process that corresponds to Glaser's stateof-the-art approach. Using community-engaged research, we aim to shift the focus from expert panels to individuals and communities with lived caregiver experiences from Indigenous perspectives. We will engage with the Indigenous communities and utilize Indigenous data analysis to develop a first-ever culturally grounded functional assessment tool in partnership with Indigenous caregivers.

Lay Abstract: We will be using community-engaged research to develop an informant based functional assessment tool to assess Instrumental Activities of Daily Living (IADL) in Indigenous population. While developing the tool, our consensus-based approach will shift the focus from expert panel to individuals and communities with lived experiences from Indigenous perspectives.

\section{CATEGORY: MASTER'S TRAINEE}

High-Resolution Diffusion Tensor Imaging of the Hippocampus Shows Differences Between

Parkinson's Disease and Healthy Controls

Alexandra Budd ${ }^{1}$, Myrlene $\mathrm{Gee}^{2}$, Krista Nelles ${ }^{2}$, Christian Beaulieu $^{3}$, Richard Camicioli ${ }^{2} .{ }^{1}$ University of Alberta; ${ }^{2}$ Department of Medicine, Division of Neurology and Neuroscience, University of Alberta; ${ }^{3}$ Department of Biomedical Engineering, University of Alberta.

Question Addressed: Do measures obtained using highresolution diffusion tensor imaging (DTI) of the hippocampus differ between patients with Parkinson's disease (PD) and healthy elderly controls? Additionally, are these measures associated with age and global cognition?

Methods: Manual hippocampal tracing was performed on novel high-resolution DTI scans in 36 individuals with PD (mean age: 68.86 years \pm 7.97 ) and 35 controls (mean age: 66.66 years \pm 6.80 ). Diffusion measures [fractional anisotropy (FA) and mean diffusivity (MD)] and global cognition [Montreal Cognitive Assessment (MoCA)] were compared across groups using independent samples t-tests. Within each group, the association between diffusion and aging as well as MoCA score was assessed using Pearson correlations.

Results: Average MoCA scores were $25.42 \pm 4.31$ for the PD group and $27.07 \pm 1.58$ for controls with available MoCA scores $(\mathrm{n}=15)$, trending towards significance $(\mathrm{p}=0.051)$. While hippocampal MD did not differ, FA was significantly lower in the PD group (mean $=0.17 \pm 0.01$ ) than in the control group (mean $=0.18 \pm 0.02 ; \mathrm{p}=0.016$ ). Age and FA were negatively correlated within the control group $(\mathrm{r}=-0.39, \mathrm{p}=0.022)$.

Research Implications: High-resolution DTI found decreased hippocampal FA between PD and control groups, suggesting 


\section{CANADIAN CONSORTIUM ON NEURODEGENERATION IN AGING (CCNA)}

that FA may be more sensitive than MD to hippocampal changes in PD. While age and global cognition were not associated with FA in PD, other factors may be linked to these hippocampal changes and should be explored.

Acknowledgements: We thank CCNA for funding this project.

Lay Abstract: The hippocampus may be affected in Parkinson's disease. This study used diffusion tensor imaging, an MRI-based method that provides indirect measures of tissue integrity, to compare the hippocampi of patients and healthy controls. One of these measures was lower in patients, suggesting decreased hippocampal integrity compared to controls.

\section{Machine Learning for the Prediction of Cognitive Decline in Parkinson's Disease}

Milton Camacho ${ }^{1}$, Hannes Almgren ${ }^{1}$, Zahinoor Ismail ${ }^{1}$, Richard Camicioli ${ }^{2}$, Oury Monchi ${ }^{1}$, Nils Forkert ${ }^{1}$. ${ }^{1}$ University of Calgary, ${ }^{2}$ University of Alberta.

Background: Parkinson's Disease (PD) is the second most common neurodegenerative disease, associated with both motor and non-motor symptoms (NMS). Various studies have highlighted the link between cognitive decline, one of the most severe NMS, and neuropsychiatric symptoms (NPS) in PD, however, their relationship is not well understood. Machine Learning (ML) is able to identify complex non-linear patterns in high-dimensional data, which can potentially identify the PD population at risk of developing dementia.

Methods: The aim was to develop ML models to classify PD, PD with Mild Cognitive Impairment (PD-MCI) and PD with dementia patients (PD-Dementia) using deep phenotyping information collected in the Canadian Consortium on Neurodegeneration in Aging COMPASS-ND study ( $\mathrm{PD}=33$ / $\mathrm{PD}-\mathrm{MCI}=22 / \mathrm{PD}-\mathrm{Dementia}=18$; Male=50/Female=23). Available data included demographics, and also cognitive, clinical, and neuropsychiatric measurements (i.e., letter fluency tests, blood pressure, and NPS, respectively). Two separate models, incorporating both a ReliefF feature selector and the random forest classifier, were developed. One model was trained using all available features and the second was trained using only demographics and NPS.

Results: The best results were achieved using all available features. A 10-fold cross-validation evaluation revealed that the model achieved $80.8 \%$ accuracy classifying individuals diagnosed with PD, PD-MCI, and PD-Dementia. The second model performed worse with $65.7 \%$ accuracy.

Conclusions: The results of this study clearly showed the benefit of multi-modal data for classification of PD patients with and without cognitive decline. Neuropsychiatric features were found to be promising when differentiating between PD and PD-Dementia, however, in this study they proved being insufficient to properly classify PD-MCI patients.
Lay Abstract: Parkinson's disease patients have a higher risk of developing dementia, however, it remains a challenge to predict their evolution. This work applied Machine learning to investigate the use of non-motor symptoms in Parkinson's disease achieving $80.8 \%$ accuracy for the identification of the Parkinson's disease population at risk of developing dementia.

\section{Investigating Changes in Cognition associated with the use of CPAP in Cognitive Impairment and Dementia: A Retrospective Study}

Yakdehikandage S. Costa, Andrew Lim, Sandra E. Black, Mark I. Boulos. University of Toronto.

Objective: To characterize the impact of 2-12 months of CPAP use on cognition in a clinical cohort with obstructive sleep apnea (OSA) and cognitive impairment due to neurodegenerative and/or vascular etiologies after controlling for baseline sleepiness.

Methods: We retrospectively analyzed 158 patients with cognitive impairment due to a neurodegenerative and/or vascular etiology and an OSA diagnosis confirmed with in-laboratory polysomnography or home sleep apnea testing (mean age $69.9 \pm 10.7 ; 69 \%$ male). Baseline Epworth Sleepiness Scores (ESS) and relevant comorbidities were obtained from selfreported questionnaires. Baseline and follow-up Montreal Cognitive Assessment (MoCA), and Mini-Mental Status Examination (MMSE) scores were obtained from clinical and research visits conducted 2-12 months apart. Adherence was defined as CPAP use $\geq 4 \mathrm{hr} /$ night, 7 days/week at followup. Associations between CPAP adherence and follow-up cognitive scores were analyzed using multivariable linear mixed-effects models.

Results: After adjusting for age, sex, body mass index, ESS, relevant comorbidities and the random effect of study cohort, CPAP adherence was significantly associated with a 2.9 point increase in follow-up MoCA scores ( $p>0.001$, $\mathrm{n}=116)$ and a 1.2 point increase in follow-up MMSE scores $(\mathrm{p}=0.03, \mathrm{n}=130)$.

Research Implications: In patients with OSA and cognitive impairment due to a neurodegenerative and/or vascular etiology, cognitive function may be stabilized or reversed with the use of CPAP. The findings of this study will aid in motivating patients to use CPAP and support future randomized controlled trials in this area.

Lay Abstract: Obstructive sleep apnea (OSA) is known to worsen cognition in patients with cognitive impairment. Our objective is to see if treating OSA by using CPAP can reverse the impact of OSA on cognition. In our clinical cohort, we demonstrate that the use of CPAP is associated with improvements in cognition. 


\section{Towards a Better Understanding of Tele-Administration Validity From Researchers' Perspectives}

Shirley Dumassais ${ }^{1}$, Gabrielle Aubin ${ }^{1}$, Karl Grewal $^{2}$, Megan O'Connell ${ }^{2}$, Natalie Phillips ${ }^{3}$, Walter Wittich ${ }^{1}$. ${ }^{1}$ University of Montreal, ${ }^{2}$ University of Saskatchewan, ${ }^{3}$ Concordia University.

Abstract: The aging population requires the consideration of the accessibility and modalities of research activities. Telehealth appears as a solution to keep research activities going in a safe way, even under the conditions of a pandemic. However, before resorting to tele-health using telephones, we must explore the feasibility of such a modality. The objective of this project is to identify the facilitators and barriers of conducting research over the phone from the perspectives of both the participants and the test administrators. Here, we report on the perspectives of researchers on conducting remote research. The pilot data of this qualitative project comprises a sample of 4 researchers $(\mathrm{MEANage}=29,75, \mathrm{SD}=12.20$, Range $=$ 23-48) who conducted telephone testing sessions with 9 older adults without sensory impairments (MEANage $=70,89$, SD $=3.59$, Range $=67-76$ ). Older adult participants completed a COVID-19 questionnaire, a telephone use questionnaire, the Montreal Cognitive Assessment screening test in its blind version and took part in a semi-structured interview. The attitude of the researchers towards remote research was explored through introspective reports after each testing session to uncover the barriers and facilitators of the phone modality. All research assistants generally reported more facilitators to conducting research over the phone than barriers. The 4 most reported themes were: testing environment, technology, personal characteristics of individuals, and communication. The findings will help future clinicians and researchers to optimize remote administration of tests and questionnaires in a way that will enhance the experience for themselves as well as their patients and/or participants.

Lay Abstract: The aging population requires the consideration of the accessibility and modalities of healthcare and research activities. However, feasibility of remote service delivery must be explored. The objective of this project is to identify the facilitators and barriers of conducting research over the phone from the perspectives of test administrators.

\section{Default-Mode Network Connectivity in Mild Cognitive Impairment: The Influence of Hearing Loss}

Nicole Grant ${ }^{1}$, Natalie Phillips ${ }^{2} .{ }^{1}$ Centre for Research on Brain, Language, and Music, Concordia University, ${ }^{2}$ Concordia University.

Abstract: Aging is associated with increases in hearing loss (HL) and cognitive decline. HL is a risk factor for dementia; however, the causal mechanisms remain unknown. The default mode network $(\mathrm{DMN})$ is a resting-state brain network that shows a pattern abnormal connectivity in both individuals with HL and mild cognitive impairment (MCI), a risk state for dementia. $\mathrm{HL}$ is prevalent in MCI, but how the co-occurrence of the two influences the DMN is unknown. We examined connectivity differences within the DMN of individuals with MCI as a function of HL. It was hypothesized that greater hearing loss would be associated with increased connectivity in the anterior DMN and decreased connectivity in the posterior DMN. Forty participants with MCI were selected form the COMPASS-ND dataset and matched on age, education, and gender; 20 had normal hearing (age $=70.23$ (5.59)) and 20 had HL (age=74.44 (4.85)). Independent components analysis was used to extract the DMN. Results indicated no significant difference in DMN connectivity across groups ( $\mathrm{p}$ $>0.05$ ), however, seed-to-voxel analyses revealed connectivity differences across groups. Individuals with HL showed decreased connectivity between both the posterior cingulate cortex and the posterior cingulate gyrus and regions of the caudate, nucleus accumbens, and thalamus. Moreover, they showed increased connectivity between both the MPFC and a lateral parietal seed region and regions of the occipital cortex. Therefore, HL was associated with reorganization within the DMN and between the DMN and regions of the occipital cortex, suggesting an overreliance on visual input or recruitment to compensate for HL.

Lay Abstract: Hearing loss (HL) is prevalent in mild cognitive impairment (MCI). We examined the effect of HL on defaultmode network (DMN) connectivity in individuals with MCI. Individuals with HL showed increased connectivity between the DMN and visual regions, suggesting the recruitment of additional brain regions or an overreliance on visual input.

\section{The Impact of the COVID-19 Pandemic on Health System Expenditures Related to Services Delivered to People Living With Dementia}

María Alejandra Rodriguez ${ }^{1}$, Isabelle Vedel ${ }^{2}$, Claire Godard-Sebillotte $^{2}$, Geneviève Arsenault-Lapierre ${ }^{2}$, Mary Henein $^{3}$, Lise Gauvin ${ }^{4}$, Nadia Sourial ${ }^{5}$. ${ }^{1}$ École de santé publique, Université de Montréal, ${ }^{2}$ Department of Family Medicine, McGill University, ${ }^{3}$ Lady Davis Institute for Medical Research, ${ }^{4}$ Department of social and preventive medicine, École de santé publique, Université de Montréal, ${ }^{5}$ Department of family and emergency medicine, Université de Montréal.

Abstract: The aging of the population creates significant challenges for the health care system, particularly for the management of people living with dementia. This vulnerable population is constantly growing, has a complex variety of health and social needs and is a major user of the health care system. In fact, expenditures related to the care of this population are five times higher than those of the population of the same age but without the disease. The COVID-19 


\section{CANADIAN CONSORTIUM ON NEURODEGENERATION IN AGING (CCNA)}

pandemic, which disrupted daily activities and access to health and social services, may have had a disproportionate impact on this population, resulting in significant changes in service utilization and its expenditure. The objective of this study is to estimate the impact of the pandemic on the use of health services and system expenditures related to these services. A quasi-experimental design comparing two groups will be used: people aged 65 years or older living at home with a diagnosis of dementia as of March 1, 2020 (exposed group) and those with a diagnosis of dementia as of March 1, 2019 (non-exposed group) in Quebec. To ensure comparability of the exposed and non-exposed groups, the inverse probability weighting method will be used. Expenditures will be calculated using an algorithm that considers the costs of each type of service. A regression model will be used to estimate the causal relation. The study will contribute to the design of effective service delivery mechanisms and public health policies that better support people living with dementia and their caregivers.

Lay Abstract: The disruption of the COVID-19 pandemic may have a disproportionate impact on people living with dementia and their related health care use and system expenditure. The focus of this study is to estimate the impact of the pandemic on health care use and system expenditure of these populations.

\section{Downregulation of Trx1 Induces Nuclear laminopathy and Contributes to the Pathophysiology of AD (winner of the Master's trainee category competition)}

Shakila Sultana, Tetiana Shcholok, Chanhyuk Jin, Md Imamul Islam, Eftekhar Eftekharpour. University of Manitoba.

Abstract: Alzheimer's disease (AD) remains the major form of dementia. Progressive neuronal loss is the major cause for the disease; however, the etiology of AD remains a controversial topic. Excessive oxidative stress, Tau hyperphosphorylation and formation of intracellular neurofibrillary tangles, as well as accumulation of extracellular Amyloid beta plaques represent the main targets for finding a cure. There is currently no effective treatment available for $\mathrm{AD}$.

Loss of cellular antioxidants including Thioredoxin-1 (Trx1) is observed in AD tissue samples. Trx 1 is a small redox protein responsible for maintaining the oxidized proteins. Examining the importance of Trx1 depletion in pathology of AD, we have previously shown that pharmacological and genetic inhibition of Trx 1 impairs autophagy. We also have identified that loss of Trx1 is an important upstream event in induction of neuronal nuclear lamina invagination. Nuclear lamina damage or laminopathy is a newly identified mechanism in pathophysiology of AD. In the current studies we use genetic inhibition of Trx1 in SH-SY5Y cells and examine the effect on nuclear events. Our results indicate that downregulation of Trx 1 is sufficient for induction of caspase 6 and induction of NL damage and expression of $\mathrm{AD}$ molecular pathology. These data are further shown in a mouse model harboring a neuron-specific Trx 1 deletion. NL damage was partially rescued in vitro conditions using a Thioredoxin mimetic peptide, CB3, (NAc-Cys-ProCys-amide). Thus, this study provides a mechanistic link between oxidative stress and pathophysiology of AD (PI is a CCNA member and this project is supported by NSERC).

Lay Abstract: Free radicals are known to cause brain cell death in dementia. Cellular antioxidants that remove these free radicals are decreased in these patients. We study how this affects the health of the brain cells and if we can find new drugs to replace the antioxidants and prevent cell death.

\section{CATEGORY: PHD AND PHD/MD TRAINEE}

\section{Towards a Better Understanding of the Montreal Cognitive Assessment Tele-Administration Validity: Preliminary Results}

Gabrielle Aubin ${ }^{1}$, Shirley Dumassais ${ }^{1}$, Karl Grewal ${ }^{2}$, Megan O'Connell ${ }^{2}$, Natalie Phillips ${ }^{3}$, Walter Wittich ${ }^{1}$. ${ }^{1}$ Université de Montréal, ${ }^{2}$ University of Saskatchewan, ${ }^{3}$ Concordia University.

Abstract: The aging of the population requires us to rethink the accessibility and modalities of healthcare services. This need is further precipitated by the COVID-19 pandemic, which drastically accentuated the workload of the already heavily burdened Canadian healthcare system. Tele-health emerges as an alternative that will facilitate the administration of healthcare services while accommodating special needs and at-risk populations. The telephone-administrated version of the Montreal Cognitive Assessment (T-MoCA; no visual items) is one of the tests that has been validated for remote use. However, the T-MoCA has yet to be validated in a visually impaired sample. The purpose of this research project is to determine the test-retest variability of the TMoCA in older adults with and without sensory impairments. This project uses repeated measures to compare the results from the in-person and over-the-phone testing sessions. The current sample consists of 10 older adults without sensory impairments (Mage $=71, \mathrm{SD}=3.4$, range $=67-76$ ), with 3 of them having completed both assessments. All participants completed a demographic questionnaire, a telephone use questionnaire, and a COVID-19 questionnaire. They provided data on the MoCA (same version in both modalities) and a 20-minute qualitative interview. As of now, 2 participants had the same MoCA score in both modalities. One participant had a difference of 3 points between both modalities, the lower score being when administered over the phone. The testing will continue until 2022, with an anticipated sample size of $n$ $=50$. We expect that the results from this project will benefit healthcare practitioners and researchers across Canada. 


\section{CANADIAN CONSORTIUM ON NEURODEGENERATION IN AGING (CCNA)}

Lay Abstract: The COVID-19 pandemic increased the need for memory tests being administered remotely. We do not know if memory tests are adapted for being done remotely. This project aims to determine if the Montreal Cognitive Assessment can reliably be administered over the phone to older adults with and without visual impairment.

\section{The Effects of an Intervention on Negative Views of Aging and Domain-Specific Self-Perceptions in Older Adults}

M. Eric Cui ${ }^{1}$, Alison Chasteen ${ }^{2}$, Maria Iankilevitch ${ }^{2}$, Niroshica Mohanathas ${ }^{3}$, April Pereira ${ }^{4}$, M. Kathleen Pichora-Fuller ${ }^{2} .{ }^{1}$ Rotman Research Institute, Baycrest, University of Toronto, ${ }^{2}$ University of Toronto, ${ }^{3} \mathrm{KITE}$ Toronto Rehabilitation Institute, University of Toronto, ${ }^{4}$ University of Waterloo.

Sensory and cognitive health are closely linked in older adults. Negative views of aging ( $\mathrm{NVoA}$ ) and domain-specific self-perceptions could predict or even affect sensory changes, cognitive performance, and overall health.

Objectives: This study aimed to investigate the effect of a two-week group educational intervention on NVoA and self-perceived abilities in sensory (hearing and vision) and cognitive domains.

Method: Participants were 166 older adults recruited from local community. Each was assigned either to the intervention group $(\mathrm{n}=83$, female $=59)$ or the control group $(\mathrm{n}=$ 83 , Female $=54)$. All participants completed hearing, vision, and cognitive screening tasks. A battery of questionnaires was completed at three different time points: pre-intervention, immediate post-intervention, and delayed post-intervention. Questionnaires measured NVoA, and self-perceptions of hearing, vision and cognition, and readiness to seek help.

Results: Preliminary multilevel models found that the general NVoA did not change, but the intervention reduced domain-specific negative attitudes and stigma, especially in participants living with corresponding impairments. Attitudes about hearing and cognition showed immediate but not delayed effects of intervention, while a gradual delayed effect of intervention was found for vision.

Conclusion: This study highlights the promising outcomes of a short intervention conducted in a research setting. The next phase of the project will investigate the possible benefits of intervention in combination with rehabilitation in clinical settings. [This study is sponsored by CCNA Team 17]

Lay Abstract: Older adults may hold negative views of health and aging. We conducted a group educational intervention focused on reducing the negative consequences of age-related stigma. This brief intervention reduced negative attitudes about age-related declines in vision, hearing, and cognition. Future research will test the intervention in clinical settings.

\section{Sex-Specific Differences in Prevalence of Cerebrovascular Disease Lesions}

Caroline Dallaire-Théroux ${ }^{1}$, Mahsa Dadar ${ }^{1}$, Colin Smith ${ }^{2}$, Richard Camicioli ${ }^{3}$, Simon Duchesne ${ }^{1} .{ }^{1}$ Université Laval, ${ }^{2}$ University of Edinburgh, ${ }^{3}$ University of Alberta.

Introduction: There are considerable and well-established sex-specific differences in terms of incidence, risk factors, manifestations, and outcomes of cerebrovascular disease (CVD). Whether these differences are also reflected in the underlying pathology remains unknown. It is therefore essential to investigate histologically whether there are sex-specific differences in prevalence of CVD lesions.

Methods: Data included 816 histopathological slides of 137 brain specimens from Edinburgh Brain Bank. After excluding individuals younger than 50 years of age and age-matching the sample, we used 558 histopathological slides for the analyses (94 brain specimens, 47 females, mean age $=79.32 \pm 9.68$, range: [53-97]). Each slide was scored (by CDT and CS) for presence of cerebral small vessel disease lesions in bilateral frontal and central white matter (WM) as well as the basal ganglia (BG). We used a series of linear and logistic regression models (with age and sex as independent variables) to assess sex-related differences in presence of arteriolosclerosis, enlarged perivascular spaces, lipohyalinosis, venous collagenosis, mediacalcinosis, cerebral amyloid angiopathy, and ischaemic and haemorrhagic lesions.

Results: Arteriolosclerosis significantly increased with age in all regions $(\mathrm{p}<0.002)$. Enlargement of perivascular spaces increased with age in bilateral frontal WM and BG. Controlling for age, women had a higher severity of arteriolosclerosis in right frontal WM, and venous collagenosis and haemorrhagic lesions in right $\mathrm{BG}(\mathrm{P}<0.05)$. We did not observe any sex-related differences in presence of lipohyalinosis, enlarged perivascular spaces, mediacalcinosis, cerebral amyloid angiopathy, and ischaemic lesions.

Conclusion: Our findings showed greater severity of CVD lesions in females than males.

Lay Abstract: In this work, we investigated whether there were sex-related differences in presence and severity of cerebrovascular disease lesions, using histopathological data of 94 aging brain specimens from Edinburgh Brain Bank. Our results showed greater severity of cerebrovascular disease lesions in females than males. 


\section{A Mixed-Methods Study on Understanding Virtual Peer Support Needs and Preferences for Maintaining Brain-Healthy Behaviours Among Older Adults}

Danielle D'Amico ${ }^{1}$, Lauren Bechard ${ }^{2}$, Samira Mehrabi ${ }^{2}$, Saara Mohamed ${ }^{2}$, Max Bergelt ${ }^{2}$, Alexandra Fiocco ${ }^{1}$, Laura Middleton ${ }^{2} .{ }^{1}$ Ryerson University, ${ }^{2}$ University of Waterloo.

Abstract: Healthy lifestyle habits (physical and cognitive activity, healthy eating, managing stress) support later life cognition and help prevent dementia, but adherence to brainhealthy lifestyle habits is low. Maintaining these habits can be challenging. Peer support can facilitate long-term lifestyle behaviour change and mitigate social isolation in older adults. The use of virtual peer support can address existing barriers to accessing in-person community programs. Although no research exists on gender differences in the use of peer support for brain health promotion in older adults, there are differences in uptake and preferences of brain-healthy lifestyle habits. Understanding gender differences in preferences, acceptability, access, and efficacy can support the development of equitable brain health promotion programs. Taking a gender lens, this study aims to understand older adults' needs and preferences for using virtual peer support to maintain brain-healthy lifestyle habits. A web-based survey and gender-specific (self-identified) virtual focus groups are being used to collect data from Canadian older adults $(\geq 60$ years) on their beliefs about brain-healthy lifestyle habits and dementia risk, current technology use behaviours, and attitudes towards using virtual peer support to maintain brain-healthy lifestyle habits. Gender differences will be investigated through gender-oriented questions in focus groups and gender-based analyses of survey data. Data collection is occurring throughout Fall 2021 with full results anticipated in Spring 2022. This interdisciplinary study addresses a gap at the intersection of gender, gerotechnology, and dementia prevention. Findings from this study will inform future virtual peer support programs to enhance later-life cognitive health in Canada's aging population.

Lay Abstract: Lifestyle habits like exercising and healthy eating can help us maintain brain health as we age, but maintaining these habits can be challenging. This study will investigate what older adults want in a virtual peer support program to maintain brain-healthy habits, which will inform development of future health promotion programs.

\section{Fostering the Inclusion of People with Dementia in Physical Rehabilitation}

Erica Dove ${ }^{1}$, Arlene J. Astell ${ }^{2} .{ }^{1}$ University of Toronto, ${ }^{2}$ KITE - Toronto Rehab Institute, University Health Network, \& University of Toronto.

Background: People with dementia are two to eight times more likely to experience a fall than older adults without dementia $^{1}$, partially due to impairments in mobility (e.g., gait) ${ }^{2}$ and balance ${ }^{3}$. Despite this, physical rehabilitation is often overlooked for people living with dementia. However, recent evidence suggests that exercise delivered through technology, such as exercise video games, can be used to improve physical function and fall risk factors among this population ${ }^{4}$.

Methods: Participants with dementia from two studies (combined $n=66$ ), both employing the same 10-week exercise intervention (Xbox Kinect bowling), were recruited from seven community-based adult day programs. The Sharpened Romberg Test (SR; $n=34$ ) and the Mini Balance Evaluations Systems Test (Mini-BEST; $n=26$ ) were conducted at baseline to assess participants' static and dynamic balance.

Results: Participants scored lower than the cut-off values on the SR (SR-EO mean: 11.3/30 sec, SR-EC mean: 3.9/30 sec) and the Mini-BEST (mean: 14.6/28 points). Despite this, among the participants who completed the Mini-BEST at baseline, only one participant (3.8\%) reported receiving previous treatment or rehabilitation for a balance impairment.

Discussion: There is a paucity of exercise interventions and physical rehabilitation geared specifically towards people living with dementia, and technology is a potential solution to offer engaging and accessible and exercise to this population. Actively including people with dementia within the scope of physical rehabilitation and exercise interventions holds implications for people with dementia themselves, as well as formal and informal caregivers, rehabilitation clinicians (e.g., physiotherapists), policymakers, researchers, and dementia support/advocacy organizations.

Lay Abstract: Physical rehabilitation is neglected for people with dementia, but research suggests that exercise can help. Participants completed an exercise intervention and balance test. Participants scored poorly on the balance test, but only one participant had received previous physical rehabilitation. Technology is a possible solution to offer exercise to this population.

\section{Does Hearing Loss and Sex Impact Training Efficacy on Dual-Task Performance in Older Adults With Mild Cognitive Impairment?}

Rachel Downey ${ }^{1}$, Niroshica Mohanathas ${ }^{2}$, Berkley Petersen ${ }^{1}$, Jennifer Campos ${ }^{2}$, Karen $\mathrm{Li}^{1} .{ }^{1}$ Concordia University, ${ }^{2}$ University of Toronto.

Abstract: Hearing loss is predictive of cognitive decline, mild cognitive impairment (MCI), and an increased risk of falling. Notably, hearing loss is more prevalent in males compared to females. One risk factor for MCI is slow dual-task walking speed. Although cognitive and physical training has been shown to improve dual-task walking speed, it is currently unclear whether hearing ability or sex affects cognitive or physical training efficacy on dual-task walking and cognitive performance in individuals with MCI. Using data from the SYNERGIC and COMPASS-ND data sets $(\mathrm{n}=57)$, we 
investigated the effect of hearing loss (normal to mild vs. moderate) and sex on changes in dual-task working memory and walking speed following combined exercise and cognitive training, exercise alone, or a control group in older adults with MCI. We found that single- and dual-task walking speed increased significantly following combined exercise and cognitive training only. The moderate hearing loss group benefited more from combined training versus exercise alone for dual-task walking speed, whereas the normal to mild hearing loss group showed no differences based on mode of training. Sex did not significantly affect training efficacy for any of the outcome measures. This study informs training recommendations for patients with MCI to help mitigate falls and cognitive decline, based on hearing ability. Specifically, our findings suggest that older adults with MCI who have poor hearing are more likely to benefit from combined cognitive and physical training, whereas older adults with normal hearing may benefit from either combined training or exercise alone.

Lay Abstract: We investigated how hearing loss and sex affect multi-tasking following exercise and cognitive training in older adults with mild cognitive impairment. Those with moderate hearing loss benefited more from combined exercise and cognitive training compared to those with normal hearing, thus informing training recommendations based on hearing ability.

\section{Memory Resilience in Cognitively Normal Older Adults with Advanced Hippocampal Atrophy: Data-Driven Approaches to Detection and Prediction (winner of the PhD and PhD/MD trainee category competition)}

Shannon M. Drouin ${ }^{1}$, G. Peggy McFall2 ${ }^{2}$ Olivier Potvin ${ }^{3}$, Lidan Zheng ${ }^{4}$, Kaarin J. Anstey ${ }^{4}$, Mario Masellis ${ }^{5}$, Pierre Bellec $^{6}$, Simon Duchesne ${ }^{7}$, Roger A. Dixon ${ }^{8}$, for the Alzheimer's Disease Neuroimaging Initiative (ADNI). ${ }^{1}$ Department of Psychology, University of Alberta, ${ }^{2}$ Department of Psychology, University of Alberta; Neuroscience and Mental Health Institute, University of Alberta, ${ }^{3}$ CERVO Brain Research Centre, Quebec, ${ }^{4}$ Neuroscience Research Australia, Sydney, Australia, ${ }^{5} \mathrm{Hurvitz}$ Brain Sciences Research Program, Sunnybrook Health Sciences Centre; Department of Medicine (Neurology), University of Toronto, ${ }^{6}$ Département d'informatique et recherche opérationnelle, Université de Montréal; Centre de recherche de l'Institut universitaire de gériatrie de Montréal, Montreal, ${ }^{7}$ CERVO Brain Research Centre, Quebec; Radiology Department, Université Laval, ${ }^{8}$ Department of Psychology, University of Alberta; Neuroscience and Mental Health Institute, University of Alberta.

Abstract: Some older adults exhibit maintained memory performance in the context of Alzheimer's disease (AD)related adversity, such as neurodegeneration or genetic risk. We investigate 'memory resilience' as a potential signal of
$\mathrm{AD}$ avoidance or pathway to healthier brain aging. The aim was to objectively classify and predict memory resilience, operationally defined as maintained memory trajectories despite longitudinal evidence of substantial hippocampal atrophy. Trajectory distributions of imaging (MRI) and memory (Rey Auditory Learning Test, ADAS - Cog, MMSE, Logical Memory) in asymptomatic adults, plus AD risk-related biomarkers, were extracted from ADNI archives $(n=365$, $\mathrm{M}$ age=74.6 [59.9-89.7], six timepoints). Focusing on left hippocampal (LHC) trajectories, we deployed data-driven longitudinal classification technologies to (1) discriminate LHC atrophy trajectory classes (2) further discriminate a memory resilient subclass, and (3) conduct machine learning biomarker prediction models of resilience. First, latent class growth models (LCGM, Mplus 8.2) identified four LHC trajectory classes. Second, LCGM applied to memory trajectories within the two most atrophied LHC classes identified a resilient subclass $(\mathrm{n}=22)$. Third, random forest analyses (sklearn, Python 3.9) tested biomarker importance in predicting memory resilience: 39 biomarkers from eight modalities were analyzed simultaneously. Twelve biomarkers from four modalities $(\mathrm{AUC}=0.69)$ explained $70 \%$ of the prediction of memory resilience. Example domains and predictors included: biological (plasma tau), demographic (education), metabolic/ vascular (pulse pressure), and co-morbidities (endocrinological medical history). The current approach elucidates the (1) complex and interactive nature of asymptomatic brain and cognitive aging and (2) pathways of memory resilience to AD adversity. Follow-up analyses will compare LHC and right hippocampal results.

Lay Abstract: We classified and predicted memory resilience, whereby non-demented older adults maintain memory performance despite elevated Alzheimer's disease risk factors. The top twelve resilience predictors were from four risk clusters: biological, demographic, metabolic/vascular, and co-morbidities. Results clarify mechanisms of resistance to neurodegeneration and pathways of healthier brain and memory aging.

\section{Diagnostic Classification \& Dementia Severity in CVLT-II-SF Forced Choice Recognition: Implications for Effort Assessment}

Karl S. Grewal ${ }^{1}$, Michaella Trites ${ }^{2}$, Megan E. O'Connell ${ }^{1}$, Debra G. Morgan ${ }^{1}$, Andrew Kirk ${ }^{1}$, Stuart W.S.

MacDonald ${ }^{2} .{ }^{1}$ University of Saskatchewan, ${ }^{2}$ University of Victoria.

Objective: Effort testing is critical to neuropsychological practice, including dementia assessment. Questions exist around whether cognitive status or impairment severity impacts effort test performance in this population. We examined whether scores on an embedded effort test - the California Verbal Learning Test II Short Form (CVLT-II-SF) Forced Choice Recognition (FCR) - differed across diagnostic groups and how severity of impairment measured with Clinical Dementia 
Rating Sum of Boxes (CDR-SOB) or mini-mental status exam (MMSE) modulated test performance.

Method: In a sample of memory clinic patients, three diagnostic groups were identified: subjective cognitive impairment $(\mathrm{SCI} ; \mathrm{n}=92)$, amnestic mild cognitive impairment (a-MCI; $\mathrm{n}$ $=18)$, and dementia due to Alzheimer's disease $(\mathrm{AD} ; \mathrm{n}=70)$.

Results: Significant group differences in FCR performance were observed using one-way ANCOVA $(\mathrm{p}<.001)$, with posthoc analysis indicating the AD group performed significantly worse than the other groups. Using multiple regression, FCR performance was modelled as a function of diagnostic group, severity (measured with either MMSE or CDR-SOB), and their interaction. Results yielded significant main effects for MMSE ( $p=0.019)$ and diagnostic group $(p=0.026)$, as well as a significant interaction $(p=0.021)$. Corresponding CDRSOB analyses were non-significant.

Conclusions: Increases in impairment disproportionately impaired FCR performance for persons with $A D$, adding caution to research-based cut scores for effort determination in dementia contexts. Caution should be used when assessing suboptimal effort in dementia populations. Future research should examine whether CVLT-II-SF-FCR is an appropriately specific inclusion in best-practice testing batteries for this population.

Lay Abstract: We wanted to see whether people with different diagnoses (Alzheimer's disease, mild cognitive impairment, and self-reported memory complaints) performed differently on a test that can help pick up faking. We found that as a person becomes more impaired, they perform worse on this test, meaning faking may be incorrectly assumed.

\section{Can Wearable Sensor Electrodermal Activity Data be Used to Detect and Monitor Neuropsychiatric Symptoms of Dementia? Preliminary Results From an Ongoing Systematic Review}

Elena Guseva ${ }^{1}$, Andrea Iaboni ${ }^{2}$, Nathan Herrmann ${ }^{3}$, Krista Lanctot $^{4}$, Dallas Seitz ${ }^{5}$, Amer M. Burhan ${ }^{6}$, Sanjeev Kumar ${ }^{7}$, Marie-Andrée Bruneau ${ }^{8}$, Andrew $\mathrm{Lim}^{4}$, Genevieve Gore ${ }^{9}$, Zahinoor Ismail ${ }^{10}$, Machelle Wilchesky ${ }^{11}$. ${ }^{1}$ Department of Family Medicine, McGill University, ${ }^{2}$ Department of Psychiatry, University of Toronto, Toronto Rehabilitation Institute, University Health Network, ${ }^{3}$ Faculty of Medicine, University of Toronto, Division of Geriatric Psychiatry, Sunnybrook Health Sciences Centre, ${ }^{4}$ Sunnybrook Health Sciences Centre, University of Toronto, ${ }^{5}$ Department of Psychiatry, Hotchkiss Brain Institute, University of Calgary, ${ }^{6}$ Ontario Shores Centre of Mental Health Science, Whitby Ontario, University of Toronto, ${ }^{7}$ Campbell Family Mental Health Research Institute, University of Toronto, ${ }^{8}$ Centre de recherche de l'IUGM (CRIUGM), Université de Montréal, ${ }^{9} \mathrm{McGill}$ University, ${ }^{10}$ Hotchkiss Brain Institute \& O'Brien Institute for Public Health, University of Calgary, ${ }^{11}$ Centre For Clinical Epidemiology Lady Davis
Institute for Medical Research - Jewish General Hospital, Department of Family Medicine McGill University.

Question Addressed: Identification of neuropsychiatric symptoms (NPS) in dementia through behavioral and psychological patterns of digitally captured physiological signals represent a tremendous potential for providing a wide range of solutions across different settings. A small but rapidly growing multidisciplinary body of evidence has investigated the validity of using wearable sensor technology (WST), revolutionizing individualized patient-centered dementia care for this population.

Methodology: We conducted a diagnostic test assessment systematic review using the Covidence systematic review web-based platform, a literature search was conducted from inception until December 2020 using Ovid MEDLINE, EMBASE, PsycINFO, EBSCOhost CINAHL, Scopus, Compendex, INSPEC, GEOBASE, IEEE Xplore library databases. Our protocol was registered https://www.crd.york.ac.uk/ prospero/display_record.php? RecordID $=219917$

Results: A systematic literature search produced 6,112 articles. Informed by the PRISMA guidelines, a final corpus of 77 titles included studies investigating the diagnostic test accuracy of actigraphy (51\%), accelerometry $(21 \%)$ and electrodermal activity (8\%). Among the 6 retained electrodermal activity (EDA) studies, 4 studies investigated sensor's ability to detect agitation. 4 studies used EDA wristbands and 2 studies were multimodal. All studies included clinical staff observations as a reference standard. Methodological quality was assessed using the QUADAS-2 tool. In the participant selection domain, we considered all studies to be at unclear risk of bias as they included small purposeful samples.

Research Implications: Preliminary evidence suggests that EDA sensors can detect states of agitation among persons living with dementia. EDA sensor data use by clinical staff may improve individualized patient-centered dementia care. EDA in combination with other sensors may provide superior results.

Lay Abstract: We reviewed 77 studies reporting on the ability of wearable devices to detect and monitor psychiatric symptoms of dementia. Results were promising among the 6 studies that used skin perspiration to detect stressful states. While more research is needed, these technologies may lead to improved dementia care in the future.

\section{Using a Machine Learning Model to Predict Older Adults' Take-Over Time in Conditionally Automated Vehicles}

Shabnam Haghzare ${ }^{1}$, Jennifer L. Campos, $\mathrm{PhD}^{2}$, Mark J. Rapoport, MD, FRCPC ${ }^{3}$, Ghazaleh Delfi, MASc${ }^{2}$, Elaine Stasiulis, $\mathrm{PhD}(\mathrm{c})^{4}$, Gary Naglie, MD, FRCPC ${ }^{4}$, Alex Mihailidis, $\mathrm{PhD}^{1} .{ }^{1}$ University of Toronto, ${ }^{2} \mathrm{KITE}$ - Toronto Rehabilitation Institute, University Health Network, ${ }^{3}$ Sunnybrook Health Sciences, ${ }^{4}$ Baycrest Health Sciences. 
Abstract: Automated Vehicles (AVs) present a potential tool for helping older adults drive safer and/or longer. However, the safety of most forthcoming AVs is conditional on their operation within certain driving conditions (Conditionally Automated Vehicles; CAVs). As such, CAVs require the drivers to take over driving control when driving conditions are outside of the CAV's operational limit. Depending on the driving conditions (e.g., speed limits), the take-over task requires varying levels of cognitive/sensory/motor resources that could be affected by age-related declines. In the current study, we developed a model to predict older adults' take-over time using cognitive/visual/motor test outcomes and driving conditions (lighting, road speed limit, road curvature). Twenty older adults were tested on a battery of cognitive, visual, and motor tests and responded to takeover requests while driving in a driving simulator. Participants' take-over performances were assessed in different lighting conditions (daytime, night-time), road speed limits $(120 \mathrm{~km} / \mathrm{h}, 60 \mathrm{~km} / \mathrm{h})$, and road curvatures (curved, straight). The proposed model was able to predict take-over with an average Adjusted R2 of 0.51 and average correlation coefficient of 0.78 . The variables of road speed, road curvature, a measure of selective attention (Useful Field of View Assessment), lighting, and a measure of working memory (WAIS Digit Span) were the five top factors respectively that contributed most to the prediction of older adults' take-over performance with the average correlation of 0.79 and adjusted $\mathrm{R} 2$ of 0.52 . The results of this study can help inform recommendations for older adults' self-regulation when using CAVs.

Lay Abstract: The state-of-the-art automated vehicles expect drivers to take over driving control when the automated vehicle can no longer drive. In this study, we propose a model that can predict the performance of healthy older drivers on this take-over tasks that can inform guidelines around automated vehicle use by older adults.

\section{Qualitative Study of Impact of Young Onset Dementia on Quality of Life of Informal Caregivers}

August Kortzman, Melanie Bayly, Megan E. O’Connell. University of Saskatchewan.

Abstract: Persons with young onset dementia (YOD) are often diagnosed only after employment, health insurance, and retirement savings have been impacted. Informal caregivers must balance sole financial responsibility with caregiving duties, which can have deleterious effect. Not much is known about the impact to caregiver's quality of life; what is known is that it is generally lower compared to caregivers of older adults with dementia. Studies have examined quality of life in caregivers of YOD as a larger construct. Conversely, we examined the subjective experience of caregivers across multiple quality of life dimensions. Qualitative analysis of seven respondents to an online survey revealed that mental, physical, and material well-being as well as self-determination, interpersonal functioning, and community connections have been impacted. Importantly, the financial consequences of YOD collapsed across multiple dimensions (not just material well-being), suggesting a global influence and a need for improved initiatives. A suggestion for further research is to compare these dimensions between caregivers of older-andyounger-onset dementia. The current research has limitations; as a small-scale study, with a limited number of respondents, saturation did not occur. Regardless, this research marks a contribution towards future targeted interventions and social policies that support persons with young onset dementia and their caregivers. Thanks to the Canadian Consortium on Neurodegeneration in Aging, the Canadian Institutes of Health Research, the Center for Aging and Brain Health Innovation, the Saskatchewan Health Research Foundation, the Alzheimer Society of Canada, and the Brain Canada Foundation for their continued support.

Lay Abstract: Caregivers of persons with young onset dementia commented on the disease's impact on their quality of life in an online survey. Common themes included lowered mental, physical, and financial wellbeing as well as poorer personal and community relationships. The findings may inform future interventions and social policy.

\section{Alterations of Neurovascular Insulin Receptor in Alzheimer's Disease}

Manon Leclerc ${ }^{1}$, Philippe Bourassa ${ }^{1}$, Cyntia Tremblay ${ }^{2}$, David A. Bennett ${ }^{3}$, Frederic Calon ${ }^{1} .{ }^{1} \mathrm{CHU}$ de Quebec, Quebec, QC, Canada, and Laval University, Quebec, QC, Canada, ${ }^{2} \mathrm{CHU}$ de Quebec, Quebec, QC, Canada, ${ }^{3}$ Rush Alzheimer's Disease Center, Rush University Medical Center, Chicago, IL, USA.

Background: The hypothesis that central insulin resistance plays a role in Alzheimer's disease (AD) is gaining interest. However, secreted insulin must first interact with the bloodbrain barrier before having an impact on brain function. In addition, $\beta$-amyloid peptides $(\mathrm{A} \beta)$ can compete with insulin binding to its insulin receptor (INSR). The aim of the present study was thus to investigate the association between INSR and $A \beta$ in brain microvessels isolated from frozen human brain samples.

Method: We used microvessel-enriched brain samples from the Religious Order Study from individuals classified as either Controls, mild cognitive impaired (MCI), or AD.

Result: The INSR was found enriched in human brain microvessels, compared to parenchymal samples. We next observed lower levels of the INSR precursor (proINSR) and INSR $\alpha-B$ in the parietal cortex of subject diagnosed with $\mathrm{AD}$, while INSR $\beta$ remained unchanged between groups. A shift toward a higher INSR $\alpha-\mathrm{A} / \mathrm{B}$ ratio was present in $\mathrm{AD}$ brain, consistent with insulin resistance. Western blot analyses showed that INSR $\alpha$-B levels positively correlated with cognitive scores. Moreover, proINSR, and mature INSR 


\section{CANADIAN CONSORTIUM ON NEURODEGENERATION IN AGING (CCNA)}

(INSR $\beta$ and INSR $\alpha-B$ ), were inversely correlated with A $\beta$ plaques in brain cortex and $\beta$-site APP cleaving enzyme 1 (BACE1) of microvessels. In addition, positive associations between Insulin-degrading enzyme (IDE) and neprilysin or p-glycoprotein (P-gp) were established.

Conclusion: Overall, our data support the hypothesis of brain insulin resistance in AD, implicating INSR localized in microvessels. Alterations of vascular INSR were associated with ante mortem cognitive impairment and with $A \beta$ or proteins involved in its production or clearance.

Lay Abstract: The vascular insulin receptor (INSR) is strongly associated with proteins involved in $\beta$-amyloid neuropathology $(\mathrm{A} \beta)$ at the blood-brain-barrier level (BBB). Using human brain samples we aim to demonstrate that INSR at the BBB is impaired in Alzheimer's disease and reflects brain insulin resistance.

\section{A Systematic Review of Community Interventions for Socially Vulnerable Older Adults}

Jasmine C. Mah ${ }^{1}$, Kenneth Rockwood ${ }^{2}$, Susan J. Stevens ${ }^{3}$, Janice M. Keefe ${ }^{3}$, Melissa K. Andrew ${ }^{4} .{ }^{1}$ Department of Medicine, Dalhousie University, Halifax, NS, Canada, ${ }^{2}$ Division of Geriatric Medicine, Dalhousie University, Halifax, NS, Canada, ${ }^{3}$ Faculty of Family Studies and Gerontology, Mount Saint Vincent University, Halifax, NS, Canada, ${ }^{4}$ Division of Geriatric Medicine, Dalhousie University, Halifax, NS, Canada.

Background: Social vulnerability occurs when individuals have been relatively disadvantaged by the social determinants of health. Interventions that reduce social vulnerability have the potential to improve health in older adults but robust evidence is lacking.

Objectives: 1) To identify, appraise and synthesize evidence on the effectiveness of interventions targeting reduction in social vulnerability for improving health related outcomes (mortality, function, cognition, subjective health and healthcare use) in older adults living in the community, and 2) to identify facilitators and barriers to implementing these interventions.

Methods: A mixed methods systematic review was conducted of primary studies using predetermined criteria in five databases and targeted grey literature. Quality was assessed using the Mixed Methods Appraisal Tool. Effectiveness data was synthesized using vote counting by direction of effect, combining $\mathrm{p}$ values and Albatross plots. Facilitators and barriers were presented as a narrative synthesis using constant comparison and thematic analyses.

Results: Across 38 included studies, there were 34 distinct interventions categorized as strengthening social supports, helping older adults and their caregivers navigate health and social services, enhancing neighbourhood environments, promoting education and providing economic stability. There was evidence to support positive influences on function, cognition, subjective health, and reduced hospital utilization. The evidence was mixed for non-hospital healthcare utilization and insufficient to determine effect on mortality. System, organizational and participant factors are also highlighted to help understand why these complex interventions achieved success.

Conclusion: Despite high heterogeneity and varying quality of studies, attention to reducing an older adult's social vulnerability assists in improving older adults' health.

Lay Abstract: Interventions that enhance social circumstances may improve health, but robust evidence is lacking. This systematic review demonstrates that providing community supports, facilitating navigation of social services, developing neighbourhood environments, promoting health literacy and providing economic stability improves function, cognition, subjective health, and reduces hospital utilization in socially vulnerable older adults.

\section{Cholinergic System Integrity as a Mechanism of Cognitive Preservation in the Oldest-Old}

Selena Maxwell ${ }^{1}$, Meghan Cash ${ }^{1}$, Sultan Darvesh ${ }^{2}$. ${ }^{1}$ Dalhousie University, ${ }^{2}$ Dalhousie University \& Mount Saint Vincent University.

Background: The oldest-old, individuals 80 years and older, have the highest prevalence of cognitive decline due to both neurodegenerative and age-related processes. A subset of this population, the cognitively normal oldest-old (CNOO), maintain cognitive function. In this study, we examine the neuropathological and cholinergic changes in the $\mathrm{CNOO}$ rostral prefrontal cortex (rPFC) and hippocampal formation to determine the contribution of cholinergic preservation in these regions to maintaining cognition in aging.

Methods: Formalin-fixed human rPFC and hippocampal tissues from male and female $\mathrm{CNOO}$ were examined and compared to sex- and age-matched Alzheimer's disease tissues (AD). The neuropathological load of these regions were examined with immunohistochemical staining for $\beta$-amyloid (A $\beta$ ), tau, $\alpha$-synuclein, and phosphorylated Tar DNA-binding protein-43 (pTDP-43). Cholinergic changes were assessed using an immunohistochemical stain for choline acetyltransferase (ChAT), and histochemical stains for acetylcholinesterase (AChE) and butyrylcholinesterase (BChE). A modified CERAD neuropathological protocol was used to analyze neuropathological findings, while ChAT activity was analyzed qualitatively.

Results: The CNOO rPFC and hippocampal formation had significant deposition of $A \beta$, tau and pTDP-43 pathology characteristic of neurodegenerative diseases. Additionally, intraneuronal $\mathrm{A} \beta$, and intraglial tau deposits were observed in the $\mathrm{CNOO}$ cases. A subset of these neuropathological aggregates were cholinesterase-positive in the $\mathrm{CNOO}$ including 
plaques, and intra- and extra-neurofibrillary tangles. ChAT activity was normal in the CNOO.

Conclusion: Despite a significant neuropathological burden, the CNOO maintain cognitive function. While cholinergic changes are present in the form of cholinesterase-associated pathology, maintained cholinergic connectivity in these regions may contribute to cognitive preservation in the $\mathrm{CNOO}$.

Lay Abstract: It is common for individuals over the age of 80 , the oldest-old, to experience a decrease in their brain's functioning, such as impaired memory and attention. This study investigates how a group of the oldest-old resist this loss of function.

\section{Older Adults' Perceptions of Ageism Before and During the COVID-19 Pandemic: Implications for Mental and Physical Health}

April Pereira $^{1}$, Maria Iankilevitch ${ }^{2}$, M. Kathleen PichoraFuller $^{2}$, Alison L. Chasteen ${ }^{2}{ }^{1}$ University of Waterloo, ${ }^{2}$ University of Toronto.

Abstract: Ageism threatens older adult's well-being, is a burden on health-care systems (Levy et al. 2020), and is widespread, with one poll showing that $82 \%$ of older adults experienced one or more forms of ageism in their everyday lives (Malani et al., 2020). Greater perceived ageism during the pandemic has been associated with lower health and life satisfaction, and continued growth has been associated with higher life satisfaction (Kornadt et al., 2021). However, it is important to take pre-pandemic levels of these variables into account to determine the extent to which these associations generalize or are pandemic-specific. In the present study, we controlled for pre-pandemic levels of the constructs to pinpoint the degree that pandemic-era experiences of ageism specifically predicted well-being. We found that higher subjective health was predicted by lower perceived ageism and higher perceived continued growth. Higher life satisfaction was predicted by higher continued growth but not ageism. There were similarities and differences in the findings of the present study compared to previous research that used only pandemic-era data (Kornadt et al., 2021). Consistent with prior research, ageism predicts subjective health and continued growth predicts life satisfaction. In contrast, we did not find that ageism predicted life satisfaction although we did find that continued growth predicted subjective health. A goal of social policy from our findings should be to address older adult's perceptions of their continued growth and aging process (Diehl et al., 2020), in addition to combatting the prevalence of ageism during both unprecedented and more usual times.

Lay Abstract: Recent research has shown that greater perceived ageism (discrimination/stereotyping based on age) during the pandemic was associated with lower reported health and life satisfaction. In the current study, we demonstrate that when including pre-pandemic scores, greater perceived ageism is only associated with lower subjective health. Other factors are also explored.

\section{Higher Angiotensin I Converting Enzyme 2 (ACE2) Expression in the Brain of Individuals With Alzheimer's Disease}

Louise Reveret ${ }^{1}$, Manon Leclerc ${ }^{1}$, Vincent Émond ${ }^{2}$, Andrée-Anne Loiselle ${ }^{2}$, Philippe Bourassa ${ }^{1}$, Cyntia Tremblay $^{2}$, David Bennett ${ }^{3}$, Sébastien Hébert ${ }^{1}$, Frédéric Calon ${ }^{1} .{ }^{1}$ Laval University, Centre de recherche du CHU de Québec, ${ }^{2}$ Centre de recherche du CHU de Québec, ${ }^{3}$ Rush University Medical Center, Chicago.

Abstract: The emergence of severe acute respiratory syndrome coronavirus 2 (SARS-CoV-2) is a major cause of death, particularly in the elderly. The geriatric population in which cognitive decline due to Alzheimer's disease (AD) is frequent is disproportionately affected by the pandemic. In addition, central nervous system (CNS) manifestations have been reported in a significant subset of SARS-CoV-2 infected patients. Since the principal entry receptor utilized by SARSCOV-2 is Angiotensin-Converting Enzyme 2 (ACE2), we examined whether ACE2 protein and mRNA levels were altered postmortem in parietal cortex samples from two different AD cohorts, totaling 142 cases. Both immunoblot and RT-qPCR analysis revealed higher concentrations of ACE2 protein and mRNA in persons with a neuropathological diagnosis of $\mathrm{AD}$, compared to age-matched controls. Brain levels of ACE2 were inversely correlated with antemortem cognitive scores. We found that ACE2 protein was highly enriched in microvessels of mice compared to brain parenchyma, but not in humans. Detachment of ACE2 from brain cell membranes was strongly associated with pericytes loss. We also found that AD patients with cerebral amyloid angiopathy (CAA) have higher ACE2 levels compared to controls with CAA. No significant change of ACE2 protein was detected in the parietal cortex from the $3 \times \mathrm{Tg}$-AD mouse model of AD neuropathology. Our data suggest that cognitive impairment is associated with higher levels of ACE2 in the brain, which might contribute the higher risk of CNS SARS-CoV-2 infection in cognitively impaired individuals and $\mathrm{AD}$ patients.

Lay Abstract: SARS-CoV-2 enters host cells via the angiotensin-converting enzyme 2 (ACE2) receptor, which is expressed in the brain, but its cellular location remains unclear. Using human brain samples, we aim to investigate the levels of ACE2 in association with clinical and neuropathological diagnosis of Alzheimer's disease. 


\section{A Systematic Review and Meta-Analysis of Multidomain Interventions in Mild Cognitive Impairment}

Talia Salzman ${ }^{1}$, Yanina Sarquis-Adamson ${ }^{2}$, Manuel Montero-Odasso ${ }^{3}$, Sarah Fraser ${ }^{4} .{ }^{1}$ School of Human Kinetics, University of Ottawa, Ottawa, ON, ${ }^{2}$ Gait and Brain Lab, Parkwood Institute, Lawson Health Research Institute, London, ON, ${ }^{3}$ Schulich School of Medicine and Dentistry, University of Western Ontario, London, ON, ${ }^{4}$ Interdisciplinary School of Health Sciences, University of Ottawa, Ottawa, ON.

Abstract: Older adults with mild cognitive impairment (MCI) experience greater cognitive declines than adults of a similar age and education level. Recent evidence has demonstrated that synergistic benefits arise from interventions that include a cognitive and physical component. This review examined whether multidomain interventions, composed of two or more different components, produced greater effects on cognition than a single intervention. Medline and Embase were systematically searched in May 2020 for studies that included: 1) objective measures of MCI; 2) non-pharmacological, multidomain interventions; 3) active control groups; and 4) randomized controlled trials. Random-effects meta-analyses were conducted on post-intervention cognitive test scores. Effect sizes were calculated from the standardized mean difference (SMD) and 95\% confidence intervals (CI). Eighteen studies evaluated multidomain interventions that targeted cognitive functions in eight domains: attention, executive function, global cognition, memory, processing speed, reaction time, verbal fluency, and visuospatial. Greater positive effects in global cognition $(\mathrm{SMD}=0.25,95 \%$ CI $0.02-0.47, \mathrm{p}=0.03$ ), memory $(\mathrm{SMD}=0.28,95 \%$ CI $0.14-0.42, \mathrm{p}<0.001)$, and verbal fluency $(\mathrm{SMD}=0.35,95 \%$ CI $0.05-0.66, \mathrm{p}=0.02)$ were observed in the multidomain group compared to the control. Multidomain interventions offer a novel, non-pharmacological strategy to improve global cognition, memory, and verbal fluency in people with MCI. Multidomain interventions may have a lesser effect on cognitive domains that remain largely intact in MCI. Intervention length varied across studies and should be further examined to determine whether cognitive effects are sustained longitudinally. This project is supported by CCNA's research Team 12: Mobility, Exercise, and Cognition.

Lay Abstract: This review found that combining two or more different types training (i.e., mental and physical) improved symptoms of mild cognitive impairment better than one training activity (i.e., physical) on its own. More research is needed to determine whether the amount of training influences how these benefits are maintained over time.

\section{Differentiation of Disease-Relevant Protein Misfolding in Alzheimer's disease Using the Fluorescent Probes BSB and MCAAD-3 and Spectral Confocal Microscopy}

Anastasiia A. Stepanchuk ${ }^{1}$, Megan L. Morgan ${ }^{1}$, Jeffrey T. Joseph ${ }^{2}$, Peter K. Stys ${ }^{1} .{ }^{1}$ Department of Clinical Neurosciences, Hotchkiss Brain Institute, University of Calgary, Calgary, Alberta, Canada, ${ }^{2}$ Department of Pathology, Cumming School of Medicine, University of Calgary, Calgary, Alberta, Canada.

Abstract: Misfolding and aggregation of proteins are prominent in most neurodegenerative diseases. For instance, accumulation of amyloid plaques and neurofibrillary tangles accompanies neuronal loss and cortical atrophy in Alzheimer's disease (AD). Despite their propensity to assemble into large beta-sheet-rich insoluble fibrils, misfolded proteins can also exist in other conformations. Evidence suggests that different prefibrillar species cause the most toxicity to the brain, whereby the mechanisms of damage are dependent on the structural assembly of misfolded proteins. While large deposits can be easily visualized using traditional histological stains, detection of smaller misfolded species has been challenging. Here, we use conformation-sensitive amyloid fluorophores BSB and MCAAD-3 that preferentially bind to and report the presence of protein aggregates via changes in their emission spectra and fluorescence intensity. Using this dye pair and spectral confocal imaging, we were able to detect different types of protein deposits in 5xFAD mouse and human AD brain sections. Double staining and spectral imaging revealed a remarkable conformational heterogeneity of amyloid pathology. Importantly, we also observed a more subtle widespread aggregate deposition in the greater brain parenchyma. Our novel paradigm allowed us to interrogate protein misfolding in $\mathrm{AD}$ in a quantitative and highly sensitive way that outperformed conventional techniques. Not only can this method be applied to further our understanding of the factors contributing to AD pathophysiology, but can also be used to study other protein misfolding disorders, within and outside of the CNS. This work was performed by the members and trainees of CCNA.

Lay Abstract: In this project, we are proposing a new method of imaging human brain tissue that revealed a more complete picture of pathological changes in Alzheimer's disease compared to conventional techniques. Our approach can be used to detect different types of improperly folded and aggregated disease-causing proteins early in disease. 


\section{CANARY: Clinical Data, Natural Language Processing, and Eye Tracking for Cognitive Risk Stratification}

Minnie Teng, Anuj Harisinghani, Matteo Rizzo, Caitlin Lewis, Hyeju Jang, Giuseppe Carenini, Cristina Conati, Dr. Thalia Field. University of British Columbia.

Abstract: Clinical trials investigating disease-modifying therapies for Alzheimer's disease (AD) are increasingly targeting participants with preclinical or early-stage neurodegeneration. Current brief screening tools are less sensitive to detecting subtle cognitive deficits in highly-educated individuals and those with early-stage disease. Other neuroimaging techniques are invasive, expensive, and cannot be administered to a widespread population. We recruited participants with clinical diagnoses of Subjective Memory Complaints (SMC), Mild Cognitive Impairment (MCI), mild to moderate AD, and healthy controls age- and sex-matched from the community. Participants completed four tasks: (1) pupil calibration, (2) picture description, (3) reading a paragraph, and (4) recalling a memory. Participant speech was recorded and eye movements were tracked using an infrared eye-tracker. Preliminary machine learning models trained using 10 -fold cross validation distinguished patients from controls with a highest AUC of $.80 \pm .02$ in an individual task alone. We then performed a late fusion analysis, combining features from all tasks, to obtain a highest AUC of $0.83 \pm 0.01$. This indicates that the four tasks are complementary to each other, as combining features from all tasks led to the highest classification accuracy. This model has demonstrated that machine learning algorithms can use speech and eye tracking data to accurately distinguish patients with early stage neurodegenerative disease from controls. Our ongoing analyses will compare remote follow up data gathered during the COVID-19 pandemic to in-person baseline data, to compare remote to in-person administration, and identify highly-predictive eye and speech features that characterize progression in neurodegeneration.

Lay Abstract: Alzheimer's disease affects millions of people worldwide. Early detection is crucial to prevent disease progression. We are using machine learning algorithms to identify people who have markers of Alzheimer's disease and cognitive impairment using speech and eye tracking data.

\section{Analysis of the HLA Locus in Isolated REM Sleep Behavior Disorder and Lewy Body Dementia}

Eric Yu ${ }^{1}$, Lynne Krohn ${ }^{1}$, Jennifer A. Ruskey ${ }^{1}$, Farnaz Asayesh $^{1}$, Sandra B. Laurent ${ }^{1}$, Dan Spiegelman ${ }^{1}$, Zalak Shah $^{2}$, Isabelle Arnulf ${ }^{3}$, Michele T.M. Hu${ }^{4}$, Jacques Y. Montplaisir $^{5}$, Jean-François Gagnon ${ }^{5}$, Alex Desautels ${ }^{5}$, Yves Dauvilliers ${ }^{6}$, Gian Luigi Gigli ${ }^{7}$, Mariarosaria Valente ${ }^{7}$, Francesco Janes ${ }^{7}$, Andrea Bernardini ${ }^{7}$, Birgit Högl ${ }^{8}$, Ambra Stefani $^{8}$, Abubaker Ibrahim ${ }^{8}$, Karel Sonka ${ }^{9}$, David Kemlink ${ }^{9}$, Wolfgang Oertel ${ }^{10}$, Annette Janzen ${ }^{10}$, Giuseppe Plazzi ${ }^{11}$, Elena Antelmi ${ }^{12}$, Michela Figorilli ${ }^{13}$, Monica Puligheddu ${ }^{12}$,
Brit Mollenhauer ${ }^{14}$, Claudia Trenkwalder ${ }^{13}$, Friederike SixelDöring ${ }^{10}$, Valérie Cochen De Cock $^{15}$, Christelle Charley Monaca $^{16}$, Anna Heidbreder ${ }^{17}$, Luigi Ferini-Strambi ${ }^{18}$, Femke Dijkstra ${ }^{20}$, Mineke Viaene ${ }^{21}$, Beatriz Abril ${ }^{22}$, Bradley F. Boeve ${ }^{23}$, Guy A. Rouleau ${ }^{1}$, Ronald B. Postuma ${ }^{1}$, The International LBD Genomics Consortium, Sonja W. Scholz ${ }^{2}$, Ziv Gan-Or' ${ }^{1}{ }^{1} \mathrm{McG}$ ill University, ${ }^{2}$ National Institute of Neurological Disorders and Stroke, ${ }^{3}$ Sorbonne University, ${ }^{4}$ University of Oxford, ${ }^{5}$ Université de Montréal, ${ }^{6}$ University of Montpellier, ${ }^{7}$ University of Udine, ${ }^{8}$ Medical University of Innsbruck, ${ }^{9}$ Charles University, ${ }^{10}$ Philipps University, ${ }^{11}$ University of Modena and Reggio-Emilia, ${ }^{12}$ University of Verona, ${ }^{13}$ University of Cagliari, ${ }^{14}$ University Medical Centre Göttingen, ${ }^{15}$ University of Montpellier IMT Mines Ales, ${ }^{16} \mathrm{CHU}$ Lille, ${ }^{17}$ University of Müenster, ${ }^{18}$ Università Vita-Salute San Raffaele, ${ }^{20}$ University Hospital Antwerp, ${ }^{21}$ St. Dimpna Regional Hospital, ${ }^{22}$ University Hospital of Nîmes, ${ }^{23}$ Mayo Clinic.

Objective: To examine genetic association of HLA alleles and amino acid for isolated REM sleep behavior disorder (iRBD) and Lewy body dementia (LBD). We compare with results in Parkinson's disease (PD).

Background: Recent studies have shown evidence for the association of HLA-DRB1 11V, 13H, 33H with PD. However, similar analysis in iRBD and LBD has not been examined in large cohorts.

Methods: We performed association studies on 1,072 iRBD cases and 918 in-house controls along with an additional 8,587 publicly available controls of European ancestry from dbGap. We also analyzed 2,604 European LBD patients and 4,032 European controls. Using HIBAG, we imputed HLA alleles and amino acid from genotyping data. Statistical analysis was performed to examine the association of specific HLA alleles and amino acid with synucleinopathies. P-value threshold were corrected for multiple testing.

Results: DRB1 37S $(\mathrm{OR}=0.76 ; 95 \% \mathrm{CI}=[-0.43,-0.11] ; \mathrm{p}=$ $8.44 * 10-4)$ is associated with iRBD. No other allele or amino acid were associated with iRBD nor LBD.

Conclusions: DRB1 37S was found to be associated with reduced risk for $\mathrm{iRBD}$. No alleles nor amino acid were found to be associated with LBD. No association to previous PD related HLA amino acids were detected in $\mathrm{RBBD}$ and LBD even though the carrier frequency is lower in cases for $\mathrm{RBD}$. This suggests that the HLA locus could play different roles in synucleinopathies. Further studies are needed to finemap the HLA locus.

Lay Abstract: We examined the association of HLA alleles, haplotypes and amino acids in two synucleinopathies: REM sleep behavior disorder (RBD) and Lewy bodies dementia. In RBD, only HLA-DRB1 37S was significantly associated with a protective effect. No alleles nor amino acids were significant for LBD. 


\section{CATEGORY: POSTDOCTORAL TRAINEE}

\section{Examining the COVID-19 Impact on People With Dementia and Their Care Partners}

Juanita-Dawne Bacsu ${ }^{1}$, Megan E. O'Connell ${ }^{1}$, Allison Cammer $^{1}$, Karl Grewal ${ }^{1}$, Shoshana Green ${ }^{1}$, Lisa Poole ${ }^{2}$, Mahsa Azizi ${ }^{1}$, Raymond J. Spiteri ${ }^{1} .{ }^{1}$ University of Saskatchewan, ${ }^{2}$ Dementia Advocacy Canada.

Abstract: The COVID-19 pandemic is taking a serious toll on people with dementia and their care partners. Research shows that dementia is the most common co-morbidity in COVID-19 related deaths. Despite this knowledge, little discussion focuses on the COVID-19 experiences of people with dementia. The purpose of this study was to understand the impact of COVID-19 on people with dementia and their care partners. Tweets were collected on Twitter using the GetOldTweets application in Python from February 15 to September 7, 2020. Filters were used to exclude irrelevant tweets $(15,737)$, and the remaining 5,063 tweets were exported to Excel for analysis. The tweets were divided among 7 coders with an additional coder managing inter-coder reliability during thematic analysis. Four main themes emerged: i) separation and loss (e.g., visitation bans, cognitive decline, and death); ii) COVID confusion, despair, and abandonment (e.g., abandonment, depression, and anxiety); iii) stress and exhaustion exacerbation (e.g., increased workload, financial challenges, social isolation, and mental health issues); and iv) unpaid sacrifices of formal care providers (e.g., inadequate PPE, limiting outside contacts, and foregoing family events). Urgent action and government leadership are needed to support people with dementia during the pandemic. Lockdown policies and lack of services have created a support vacuum, leaving people with dementia and their care partners at a point of crisis. In developing COVID-19 policies and programs to support people with dementia and their care partners, there is an imminent need for collaborative research and co-creation methods to ensure maximum impact.

Lay Abstract: Using Twitter, our study examined the impact of COVID-19 on people with dementia. We found several challenges including separation from family, feelings of loss and hopelessness, and increased workloads and stress for care partners. Government leadership and collaborative research are urgently needed to support people with dementia during the pandemic.

\section{The Psychological and Social Wellbeing of Older Adults Living Alone With Cognitive Impairment (winner of the Postdoctoral trainee category competition)}

Melanie Bayly, Megan E. O’Connell, Debra Morgan. University of Saskatchewan.

Background: Research suggests older adults living alone are at higher risk for poorer psychological and social wellbeing, and those with dementia report high levels of unmet needs, social isolation, and loneliness. The current project, for which the first author was funded as a trainee through CCNA, extends this literature to examine relationships between wellbeing, living situation, and the broader spectrum of cognitive impairment (CI).

Methods: Survey data were obtained from the Canadian Longitudinal Study on Aging (wave 1). From 21,241 "tracking" participants, we used an algorithm developed by the second author to identify those with CI. Using profile analysis, we compared three groups of older adults $(60+)$ on indices of psychological wellbeing (depressive symptoms, perceived mental health, satisfaction with life) and social wellbeing (social support, loneliness, community participation, and social contact): those with CI living with others $(\mathrm{n}=210)$, those living alone with $\mathrm{CI}(\mathrm{n}=123)$, and a matched group living alone without $\mathrm{CI}(\mathrm{n}=123)$.

Results: Those living alone with CI reported poorer wellbeing than both other groups on all measures other than loneliness (no different than the other group living alone) and community participation, social contact, and perceived mental health (no different than the other group with CI). Further, sex-stratified analyses suggest that living alone may be associated with poorer wellbeing for males, particularly those with CI.

Implications: Findings suggest that those living alone with CI should be considered an at-risk group for psychosocial wellbeing who may benefit from additional supports, even without a dementia diagnosis.

Lay Abstract: We examined whether older adults living alone with cognitive impairment (CI) are at unique risk for poor psychological and social wellbeing. Findings showed this group consistently reported lower wellbeing compared to those living alone without CI and those with CI living with others, and may benefit from supports. 


\section{High-Level Cognition And Imaging Biomarkers In A Mouse Model Of Dementia Due To Leukoencephalopathy With CSF1R Haploinsufficiency}

Daniel Menezes Guimarães ${ }^{1}$, Aja Elizabeth Hogan-Cann ${ }^{1}$, Kate M. Onuska ${ }^{1}$, Chris Fodor ${ }^{1}$, Matthew F. Cohan ${ }^{1}$, Vania F. Prado $^{2}$, Jane Rylett ${ }^{3}$, Lisa M. Saksida ${ }^{4}$, Taylor W. Schmitz ${ }^{3}$, Elizabeth Finger ${ }^{5}$, Robert Bartha ${ }^{6}$, Timothy J. Bussey ${ }^{4}$, Marco A. M. Prado ${ }^{7} .{ }^{1}$ Robarts Research Institute, The University of Western Ontario, London, Ontario, Canada, ${ }^{2}$ Robarts Research Institute, The University of Western Ontario, London, Ontario, Canada. Department of Physiology and Pharmacology, The University of Western Ontario, London, Ontario, Canada. Department of Anatomy \& Cell Biology, The University of Western Ontario, London, Ontario, Canada,

${ }^{3}$ Robarts Research Institute, The University of Western Ontario, London, Ontario, Canada. Department of Physiology and Pharmacology, The University of Western Ontario, London, Ontario, Canada, ${ }^{4}$ Robarts Research Institute, The University of Western Ontario, London, Ontario, Canada. Department of Physiology and Pharmacology, The University of Western Ontario, London, Ontario, Canada. BrainsCAN, The University of Western Ontario, London, Ontario, Canada, ${ }^{5}$ Robarts Research Institute, The University of Western Ontario, London, Ontario, Canada. Department of Clinical Neurological Sciences, The University of Western Ontario, London, Ontario, Canada, ${ }^{6}$ Robarts Research Institute, The University of Western Ontario, London, Ontario, Canada. Department of Medical Biophysics, The University of Western Ontario, London, Ontario, Canada, ${ }^{7}$ Robarts Research Institute, The University of Western Ontario, London, Ontario, Canada. Department of Physiology and Pharmacology, The University of Western Ontario, London, Ontario, Canada. Department of Anatomy \& Cell Biology, The University of Western Ontario, London, Ontario, Canada.

Abstract: Adult-onset leukoencephalopathy with axonal spheroids and pigmented glia (ALSP) is a rare condition of unknown prevalence and which has no cure. It is characterized by white matter degeneration, early dementia, psychiatric and cognitive symptoms. The array of nonspecific symptoms enhances the frequency of misdiagnosis. ALSP is an autosomal dominant disease commonly caused by loss-of-function mutations in the colony-stimulating factor- 1 receptor gene (CSF1R), which is mainly expressed in microglia. Given the small number of patients, a murine model and robust phenotyping pipeline are needed to define therapeutic targets. Using heterozygous knockout mice for CSF1R, we investigated high-level cognition with touchscreen technology, employed diffusion tensor magnetic resonance imaging, positron emission tomography with a TPSO ligand for neuroinflammation, and biochemical techniques to characterize parenchyma alterations, both at the anatomical and cellular levels. Using a comprehensive longitudinal design to probe different cognitive skills (e.g., cognitive flexibility and attention), we observed sex-specific phenotypes. While male heterozygous mice showed lower attentional/impulse control performance and an increased difficulty to extinguish learned behavior, females showed visuospatial learning and behavioral flexibility impairments. In addition, behavior impairments were detected earlier in females (7-10 months) than in males (14-19 months). In males, cognitive changes were accompanied by neurodegeneration of the posterior parietal cortex, whereas in females, increased neuroinflammation was detected using PET imaging. This work demonstrates the potential of a multimodal imaging and multifactor cognitive profiling pipeline for testing symptomatic and disease-modifying therapies that could be translated for treatment of ALSP.

Supported by: Canadian Consortium on Neurodegeneration in Aging and BrainsCAN

Lay Abstract: Adult-onset leukoencephalopathy with axonal spheroids and pigmented glia (ALSP) causes early-onset dementia and has no cure. Identifying faithful animal models is an important step to test new therapeutical approaches. We developed a pipeline for imaging and high-level cognition to test new therapeutics for ALSP in mice.

\section{Progression of Neuropsychiatric Symptoms in Pre- Dementia GRN and C9orf72 Mutation Carriers}

Hyunwoo Lee ${ }^{1}$, Atri Chatterjee ${ }^{1}$, Dana Wittenberg ${ }^{1}$, Rosa Rademakers ${ }^{2}$, Ian R Mackenzie ${ }^{3}$, Veronica HirschReinshagen ${ }^{3}$, Ging-Yuek Robin Hsiung ${ }^{1} .{ }^{1}$ Division of Neurology, Department of Medicine, University of British Columbia, Canada, ${ }^{2}$ Department of Neuroscience, Mayo Clinic, Jacksonville, FL, USA, ${ }^{3}$ Department of Pathology and Laboratory Medicine, Faculty of Medicine, University of British Columbia, Canada.

Background: Neuropsychiatric symptoms (NPS) are common in frontotemporal dementia (FTD) patients and may precede the clinical diagnosis of dementia. We hypothesized that GRN and C9orf72 mutation carriers have increased baseline burden and rates of progression of NPS prior to the onset of dementia.

Method: We analyzed longitudinal data from 80 participants recruited through the UBC FTD Study. (21 C9orf72+ mutation carriers, $10 \mathrm{GRN}+$ carriers, and 49 non-carrier family members; mean \pm SD age in years: $47 \pm 13,51 \pm 9$, and $54 \pm 13$, respectively) NPS were assessed annually with Neuropsychiatric Inventory Questionnaire (NPI), Frontal Behavioural Inventory (FBI), Beck Depression Inventory (BDI) and Iowa Scale of Personality Change (ISPC) with a mean followup of 5.8 years. If a participant developed FTD during the follow-up $(\mathrm{N}=2)$, we only analyzed observations collected before the conversion. We compared the baseline burden of NPI, FBI, BDI and ISPC adjusted for age and sex, among C9orf72+ carriers, GRN + carriers and non-carriers. We then used random-intercept mixed models to compare the rates of progression of NPS among genetic groups. 


\section{CANADIAN CONSORTIUM ON NEURODEGENERATION IN AGING (CCNA)}

Result: There were no significant group differences in baseline demographic features or neuropsychiatric measures. In longitudinal assessments, non-carriers demonstrated minimal change in NPS measures. GRN+ carriers had higher progression rates of ISPC compared to non-carriers. C9orf72+ carriers showed decline in BDI scores over time, compared to non-carriers.

Conclusion: Our findings suggest that NPS may reflect very early brain changes in FTD. ISPC may be more sensitive to early changes in FTD compared to instruments like FBI, which are helpful after the onset of dementia.

Lay Abstract: People with frontotemporal dementia (FTD) often experience mental or emotional disturbances (neuropsychiatric and behavioral symptoms) such as changes in personality or feeling depressed. These disturbances may begin a long time before people actually get diagnosed with FTD.

\section{Nutrition Risk and Its Association with Dementia Diagnosis and Severity in Older Adults Referred to A Rural and Remote Memory Clinic}

Elham Movassagh ${ }^{1}$, Debra Morgan ${ }^{1}$, Julie Kosteniuk ${ }^{1}$, Megan O'Connell2, Andrew Kirk ${ }^{3}$, Allison Cammer', Chandima Karunanayake ${ }^{1} .{ }^{1}$ Canadian Centre for Health and Safety in Agriculture, University of Saskatchewan, ${ }^{2}$ College of Arts and Science, University of Saskatchewan, ${ }^{3}$ College of Medicine, University of Saskatchewan, ${ }^{4}$ College of Pharmacy and Nutrition, University of Saskatchewan.

Objectives: To assess undernutrition risk using SCREEN II (Seniors in the Community: Risk Evaluation for Eating and Nutrition) and to evaluate the association between undernutrition risk and dementia diagnosis and severity.

Methods: The study included 167 (60\% females) communitydwelling older adults (aged $x x>55$ ) from rural and remote areas of Saskatchewan referred to a Rural and Remote Memory Clinic. Nutritional risk was determined by SCREEN II scores. Dementia diagnosis was ascertained by interdisciplinary team examination. The Mini-Mental State Examination (MMSE) and the Clinical Dementia Rating-Sum of Boxes (CDR-SOB) were used to measure dementia severity. We used logistic regression models to estimate association between nutritional risk and dementia diagnosis and severity.

Results: The mean age of participants was $71.1 \pm 8.7$ years. Compared to men, a higher proportion of women were at a high nutritional risk (60\% vs. 55.2\%) and/or had dementia ( $57 \%$ vs. $38 \%$ ) with a more severe loss of cognitive function compared to men. Men had greater BMI and comorbidity than women. The most prevalent risk factors for men and women were inadequate intake of milk, milk products, fruits, vegetables, and fluids, and perception of own weight. Type of dementia diagnosis and severity were not associated with nutritional risk, even after adjusting for sex, age, BMI, comorbidity, education, and metropolitan influence zone.

Conclusion: More than half of the study population were at high nutritional risk. Dementia diagnosis or severity was not associated with higher nutrition risk. Early detection of nutritional risk is essential to implement appropriate interventions and prevent adverse outcomes.

Lay Abstract: We evaluated the undernutrition risk factors in older adults who were admitted to a memory clinic. More than half of the participants were at high nutritional risk group, with women being more at risk than men. There was no association between dementia and severity of cognitive decline and undernutrition risk.

\section{Cardiovascular Risk Factors and Risk of Alzheimer's Disease and Death: A Latent Class Approach}

Myuri Ruthirakuhan ${ }^{1}$, Hugo Cogo-Moreira ${ }^{2}$, Walter Swardfager ${ }^{1}$, Nathan Herrmann ${ }^{1}$, Krista L. Lanctot ${ }^{1}$, Sandra E. Black ${ }^{1} .{ }^{1}$ Sunnybrook Research Institute, Toronto, ON, ${ }^{2}$ Østfold University College, Halden, Norway.

Objective: Cardiovascular risk factors (CVRFs) co-occur with one another and little is known regarding the extent of their clustering, and risk of Alzheimer's disease (AD). We identify CVRF phenotypic classes in cognitively normal $(\mathrm{CN})$ individuals, and investigate between-group differences in incident $\mathrm{AD}$ and mortality.

Methods: $\mathrm{CN}$ individuals were recruited from the National Alzheimer's Coordinator Center. A latent class analysis was conducted with seven CVRFs (hypertension, hypercholesterolemia, heart condition, stroke, smoking, diabetes, and high body mass index (BMI)). Between-group differences in incident $\mathrm{AD}$, and mortality were investigated. Post-hoc analyses investigated differences in death without progression to $\mathrm{AD}$, incident $\mathrm{AD}$ in those who remained alive, and age of death.

Results: This study included 12,412 CN individuals (average follow-up: 65 months). Three CVRF phenotypes were identified: 1) no CVRFs (reference) $(\mathrm{N}=5398(43 \%)), 2)$ hypertension and hypercholesterolemia (vascular-dominant) $(\mathrm{N}=5721(46 \%)$ ), and 3) hypertension, hypercholesterolemia, diabetes, and high BMI (vascular/metabolic) $(\mathrm{N}=1293(10 \%))$. Only the vascular-dominant phenotype had a greater incidence of $\mathrm{AD}(\mathrm{OR}: 1.30, \mathrm{p}<.001)$. Mortality was greater in both the vascular-dominant (OR:3.26,p $<.001)$, and vascular/metabolic phenotypes (OR:1.84,p=.02). Incidence of death without AD was greater in the vascular-dominant (OR: $3.31, \mathrm{p}<.001)$ and vascular/metabolic phenotypes (OR:3.12,p<.001). Incident $\mathrm{AD}$ in those who remained alive was greater in the vasculardominant (OR:1.54, $\mathrm{p}=.02)$ and vascular/metabolic phenotypes (OR:1.46,p=.04). Only the vascular/metabolic phenotype had a significantly younger age of death compared to the reference group (84.3 vs. 88.7 years, $p<.001)$. 


\section{CANADIAN CONSORTIUM ON NEURODEGENERATION IN AGING (CCNA)}

Interpretation: Three CVRF phenotypes were identified in $\mathrm{CN}$ elderly. Only the vascular-dominant phenotype was associated with a greater incidence of AD. Selective mortality may contribute to the attenuated association between the vascular/metabolic phenotype and incident $\mathrm{AD}$.

Lay Abstract: In a sample of 12,412 elderly individuals who do not have memory problems, we studied how cardiovascular risk factors, such as high blood pressure and diabetes, occur with one another, and how groups of these risk factors are associated with risk of Alzheimer's disease and death.

\section{CIRCA-CA: A Digital Solution for People With Dementia, During and After COVID-19}

Danielle Saney, Erica Dove, Mary Hynes, Sukriti Sachdev, and Arlene Astell. University of Toronto, KITE - Toronto Rehabilitation Institute

Background: The Computer Interactive Reminiscence and Conversation Aid (CIRCA) is a multimedia conversation support for people living with dementia and caregivers. Originally developed in the UK, this project will customize CIRCA for the Canadian population, to create CIRCA-CA. The project addresses the reduced opportunities that people living with dementia have for social interaction and stimulation created by the COVID-19 lockdown and physical distancing.

Methods: In study Phase 1, a series of interactive co-creation sessions with older adults will be held throughout Canada. Sessions will focus on allowing participants to discuss their lived experiences, important cultural, historical events and the types of content that they would like to see as part of CIRCA. Once CIRCA is populated with the new contents based on the findings of these sessions, the second phase will involve implementing CIRCA-CA in community organizations and specialized dementia care units. The sessions will inform an evaluation framework for facilitating co-creation with older adults and furthermore, how to best implement a new program. Ongoing support and updating of contents will be available as part of the sustainability plan.

Results and Discussion: CIRCA-CA can provide conversation and meaningful occupation to benefit people living with dementia and their families, thereby tackling the stress and burden created through lack of programming and contacts during the COVID-19 pandemic. Developing content for representation of various cultures and experiences facilitates inclusivity and a sense of community.

Lay Abstract: We are working alongside older adults to add Canadian information to CIRCA, an electronic scrapbook. Participants are given the opportunity to discuss personal history and experiences which will help to form the new content. Using CIRCA helps persons with dementia, their families, friends, and caregivers talk about past memories together.

\section{Central Nervous System Active Medications in Older Adults With Dementia: Sex Differences and Polypharmacy}

Shanna C. Trenaman ${ }^{1}$, Susan K. Bowles ${ }^{2}$, Susan Kirkland ${ }^{3}$, Melissa K. Andrew ${ }^{4}$. ${ }^{1}$ Dalhousie University, Department of Medicine, ${ }^{2}$ Nova Scotia Health; Dalhousie University, College of Pharmacy, ${ }^{3}$ Dalhousie University, Department of Community Health and Epidemiology, ${ }^{4}$ Dalhousie University, Department of Medicine; Nova Scotia Health.

Objectives: To describe central nervous system (CNS) active medication use in a cohort of older adults with dementia in Nova Scotia, Canada, including a sex-disaggregated analysis.

Methods: Retrospective cohort study of those aged 65 years or older with recorded dementia between 2005 and 2015. Medication dispensation data was collected from either April 1, 2010 or dementia diagnosis (cohort entry) to either March 31, 2015 or death. CNS-active medication claims were reported in each of five fiscal years with an overall summary. Antipsychotics, benzodiazepines, nonbenzodiazepine benzodiazepine receptor agonists, and most antidepressants were captured. Medication use, sex, age, and rurality were described. Odds ratios provided sex comparisons and risk factors associated with specific CNS-active medication use.

Results: The cohort included 28,952 adults with dementia (median age 82.0 years; $62.0 \%$ female), with $54.3 \%(\mathrm{n}=$ $15,728)$ receiving at least one CNS-active medication. Nearly a quarter $(23.8 \%)$ were dispensed three or more CNS-active medications. Those exposed to CNS-active medications were younger (median age, 81 versus 83 years, OR: 0.98, $95 \% \mathrm{CI}[0.97,0.98]$ ), more likely to be women (OR: 1.80 , $95 \% \mathrm{CI}[1.72,1.88])$, and less likely to be in a urban location (OR: $0.71,95 \% \mathrm{CI}[0.68,0.75])$ than those not exposed. Antidepressants were the most dispensed class, but quetiapine the most dispensed medication. Women were more likely dispensed antidepressants or sedatives and men first generation antipsychotics.

Conclusions: This study raises concerns that prescribers assume younger adults with dementia can tolerate greater CNS-active medication burdens and that rural locations may need increased access to non-pharmacological options for dementia management.

Lay Abstract: Older adults with dementia may be prescribed one or more central nervous system (CNS) active medications to manage their symptoms of dementia. We identified that multiple CNS-active medications are commonly prescribed and that there are differences in CNS-active medication use for older men compared to women with dementia. 